\title{
Emotional and Behavioural Problems among Sheltered Homeless Children
}

\author{
Saroj Prasad Ojha, 'Jasmin Ma, ${ }^{1}$ Manisha Chapagain, ${ }^{1}$ Pratiksha Tulachan ${ }^{1}$ \\ 'Department of Psychiatry and Mental Health, Maharajguni Medical Campus, Tribhubhan University Teaching Hospital, \\ Kathmandu, Nepal.
}

\section{ABSTRACT}

Introduction: Homelessness in children is a serious problem in the society. Factors leading to homelessness in Nepalese children are poverty, unemployment, multiple children, exposure to violence, caste based social discrimination etc. Children living out of their homes have problems in attachment, temperament, social skills, cognitive ability, and language development. Few nongovernmental organizations that have started to look after these children but the mental health need is neglected. The study attempted to investigate the emotional and behavioural problems in homeless children in one of the shelters in Kathmandu.

Methods: All the children $(n=126)$ in the SAATHI Organization were included in the study. They were administered Child Behaviour Check List 6-18. Children with T-score $\geq 65$ on externalising or internalising dimension were further evaluated in the Child Guidance Clinic for the final diagnosis based on DSM IV.

Results: The prevalence of emotional and behavioural problem among the participants was 36 (28.57\%) [15 (26.31\%) in boys and 21 (30.43\%) in girls] based on CBCL/6-18 years and 29 (23.01\%) [12 $(21.05 \%)$ in boys and $17(24.63 \%)$ in girls] based on final diagnosis. The conduct problems was the most common among the male children 5 (8.77\%), followed by ODD 3 (5.26\%), ADHD 2 (3.5\%), Anxiety $2(3.50 \%)$ whereas Anxiety is most common in female children $9(13.04 \%)$, followed by depression $5(7.24 \%)$, conduct $3(4.34 \%)$.

Conclusions: The rate of emotional and behavioral disorder in homeless children in the study is similar to the school aged non homeless children in previous studies, which may be because of the protection provided by the shelters. This study highlighted the need for methodologically better studies in the field.

Keywords: children; emotional and behavioural problems; homelessness; Nepal.

\section{INTRODUCTION}

World Health Organization statistics reveal that the prevalence of disabling mental illnesses among children and adolescence attending health care centres range between $20-30 \%$ in urban areas and $13-18 \%$ in rural areas. $^{1}$

More than 3 million people each year experience homelessness which includes 1.3 million children. ${ }^{2}$
There are no statistics regarding the Homeless children in Nepal. There are many possible reasons for the children to be homeless in Nepal, the reason beingarmed conflict, poverty, unemployment, caste based discrimination, etc. Anxiety, depression, anger

Correspondence: Dr. Saroj Prasad Ojha, Department of Psychiatry and Mental Health, Maharaiguni Medical Campus, Tribhuvan University Teaching Hospital, Kathmandu, Nepal. Email: ojhasp@yahoo.com, Phone: 9851038299 
are common feelings which are often seen in behavior these homeless children may exhibit. ${ }^{3}$

Attempt to provide food, shelter and other needs like education by the private sector is highly appreciable. Still, the mental health of homeless children is not adequately taken care of. We have attempted to assess the emotional and behavioural problems in the sheltered homeless children and adolescents in one of the shelters in Kathmandu.

\section{METHODS}

A cross-sectional observational study was conducted in 2012. The participants included 126 male and female homeless children. These children were recruited from one of the foster homes helping homeless children in Kathmandu. The shelter was selected on the basis of convenience. The shelter recruits children from all over Nepal. The mostly recruited children were orphans, abused (physically and sexually), neglected by the parents, belonging to poor family, children from the streets or displaced during the decade long armed conflict. However, the institution denies children with obvious psychiatric problems including mental retardation. These children were kept in four separate houses based on their age. Those under 12 years were kept in junior section and those above 12 were staying in the senior section and males and females were kept separately. The institution provides food, shelter, education, health care to these children for free. All the children between 6 to 18 years in the shelter were included in the study excluding those who cannot make communication, those with intellectual disabilities or those requiring urgent medical evaluation.

The informed consent was taken from the respective wardens of the shelters. This study was conducted in two stages. The participants were screened with Child Behaviour Checklist 6-18 years (CBCL/6-18) in the first stage. The $\mathrm{CBCL}$ was reported by the hostel warden and psychologist in respective hostels, who were instructed about the tool before. Thus obtained CBCL form for each child was entered into the computer program Assessment Data Manager. The ADM presented the interpretation in different categories. Only the DSM IV oriented scales were further analysed. DSM-oriented scales comprise six items like Affective problems, Anxiety, Somatic problems, Attention Deficit Hyperactivity problem, Oppositional Defiant Problems, and Conduct Problems. This questionnaire (CBCL) was used as a screening instrument to identify children at high risk for psychiatric disorder. All children scoring above the normative cut off point i.e. $T$ scores in borderline (65-69) and clinical range (>70) were recruited for detail psychiatric assessment, the second stage procedure.
The second-stage clinical assessment was carried out by using semi-structured proforma and diagnoses were made on the basis of clinical interview by using DSM IV TR criteria. The final diagnosis was made in consultation with the psychiatrist in Child Guidance Clinic in TUTH, Maharajgunj. Although, the investigator was aware of the study design and methodology, s/ he was blinded to the scoring results of the first-stage on the individual child who was being evaluated. At the end all the children requiring clinical attention were further managed in the hospital with both psychiatric and clinical-psychological services.

Ethical clearance is taken from the Ethical Review Board of our institute and there are no harms to the participants in the study.

Data was described in terms of mean, standard deviation and percentages. Bi-variate analysis for categorical variables was performed using Chi square test. For parametric data, independent sample t test was used. Significance was set at $5 \%$.

\section{RESULTS}

(Table 1 ) shows that out of total participants $(n=126)$, female participants $69(54.8 \%)$ outnumbered the males $57(45.2 \%)$. The majority of the participants were from 11 to 15 years age group $77(61.12 \%)$, with mean (SD) age of the participants being 12.16 (3.00) years. The predominant participants $(35.7 \%)$ were of ethnic minorities (Mongolians like Gurung, Magar, Tamang, etc).

\begin{tabular}{|lll|}
\hline $\begin{array}{l}\text { Table 1. Socio demographic } \\
\text { participants (N=126). }\end{array}$ & Number & Percentage \\
\hline Characteristic & & \\
Gender & 57 & $45.2 \%$ \\
Male & 69 & $54.8 \%$ \\
Female & & \\
Age group (Years) & 34 & $26.98 \%$ \\
$6-10$ & 77 & $61.12 \%$ \\
$11-15$ & 15 & $11.90 \%$ \\
$16-18$ & & \\
Ethnicity & 19 & $15.1 \%$ \\
Brahmin & 42 & $33.3 \%$ \\
Chhetri & 5 & $4.0 \%$ \\
Newar & 45 & $35.7 \%$ \\
Gurung/Tamang/Magar & 15 & $11.9 \%$ \\
Dalit & & \\
Mean (SD) Age of the & & \\
participants (years): 12.16 & & \\
(3.00) & & \\
\hline
\end{tabular}


Ojha et al. Emotional and Behavioural Problems among Sheltered Homeless Children

(Table 2) shows that females have significantly high mean $T$ score than males on affective [62.13 vs 54.61], somatic [59.54 vs 52.30], anxiety [58.59 vs 55.37], ADHD [55.67 vs 52.23] and conduct [60.64 vs 57.23]. Though, $T$ scores on ODD are higher in females the observed difference is not significant.

\begin{tabular}{|llll|}
\hline \multicolumn{4}{|c|}{ Table 2. T Score Mean (SD) according to gender. } \\
\hline & Male & Female & p value \\
T affective & $54.61(4.34)$ & $62.13(7.90)$ & $<0.001$ \\
T anxiety & $55.37(7.56)$ & $58.59(8.10)$ & 0.024 \\
T somatic & $52.30(4.55)$ & $59.54(5.05)$ & $<0.001$ \\
T ADHD & $52.23(4.78)$ & $55.67(5.83)$ & $<0.001$ \\
T ODD & $55.21(7.52)$ & $56.09(6.20)$ & 0.475 \\
T Conduct & $57.23(7.75)$ & $60.64(7.88)$ & 0.016 \\
\hline
\end{tabular}

${ }^{*}$ Chi Square Test

* p value may not carry any statistical significance as the male value for clinical score is zero Table 3 shows the distribution of different categories of $\mathrm{T}$ score like clinical range, borderline range normal range based on gender. The observed difference is significant only for ODD score, where male children have higher score $(p=0.038)$. The observed difference in affective and somatic score may be due to no males with clinical range score. T score on anxiety (clinical range) is higher in females though not significant and ADHD T score (clinical range) is higher in males.

Table 3. T score categories according to gender $\mathbf{n}(\%)$.

\begin{tabular}{|c|c|c|c|}
\hline T affective & Male & Female & $\mathbf{P}$ value* \\
\hline$\geq 70$ (clinical) & 0 & $\begin{array}{l}14 \\
(20.3)\end{array}$ & $<0.001 * *$ \\
\hline 65-69(borderline) & $2(3.5)$ & $\begin{array}{l}13 \\
(18.8)\end{array}$ & \\
\hline$<65$ (normal) & $55(96.5)$ & $\begin{array}{l}42 \\
(60.9)\end{array}$ & \\
\hline \multicolumn{4}{|l|}{$T$ anxiety } \\
\hline$\geq 70$ (clinical) & $5(8.8)$ & $8(11.6)$ & 0.365 \\
\hline $65-69$ (borderline) & $7(12.3)$ & $\begin{array}{l}12 \\
(17.4)\end{array}$ & \\
\hline$<65$ (normal) & 45 (78.9) & $49(71.0)$ & \\
\hline \multicolumn{4}{|l|}{ T somatic } \\
\hline$\geq 70$ (clinical) & 0 & $2(2.9)$ & $0.003 * *$ \\
\hline 65-69(borderline) & $1(1.8)$ & $11(15.9)$ & \\
\hline$<65$ (normal) & $56(98.2)$ & $\begin{array}{l}56 \\
(81.2)\end{array}$ & \\
\hline \multicolumn{4}{|l|}{ T ADHD } \\
\hline$\geq 70$ (clinical) & $2(3.5)$ & $1(1.4)$ & 0.877 \\
\hline 65-69 (borderline) & $2(3.5)$ & $6(8.7)$ & \\
\hline
\end{tabular}

\begin{tabular}{|llll|}
$<65$ (normal) & $53(93.0)$ & 62 (89.9) & \\
T ODD & & & \\
$\geq 70$ (clinical) & $4(7.0)$ & $1(1.4)$ & 0.038 \\
$65-69$ (borderline) & $9(15.8)$ & $6(8.7)$ & \\
$<65$ (normal) & $44(77.2)$ & $62(89.9)$ & \\
T Conduct & & & \\
$\geq 70$ (clinical) & $8(14.0)$ & $8(11.6)$ & 0.069 \\
$65-69$ (borderline) & $3(5.3)$ & $23(33.3)$ & \\
$<65$ (normal) & $46(80.7)$ & $38(55.1)$ & \\
\hline
\end{tabular}

(Table 4) shows that depression ( $p=0.008)$ and anxiety disorder $(\mathrm{P}<0.001)$ were significantly higher in children of more than 10 years. However other problems like somatic, ADHD, ODD and Conduct were not different between the age group. As shown in table $5,29(23.01 \%)$ had some of the clinically diagnosable emotional and behavioural disorder.

\begin{tabular}{|llll|}
\hline \multicolumn{4}{|c|}{ Table 4. Distribution of T scores by age group (years). } \\
\hline \multicolumn{4}{|c|}{ Age group (years), Mean(SD) } \\
& $<10$ years & $\geq 10$ years & p value* \\
T affect & $55.57(5.22)$ & $59.36(7.76)$ & 0.008 \\
T anxiety & $52.67(4.93)$ & $58.03(8.21)$ & $<0.001$ \\
T somatic & $57.57(5.05)$ & $56(6.18)$ & 0.277 \\
T ADHD & $52.43(4.26)$ & $54.45(5.82)$ & 0.072 \\
T ODD & $53.95(6.70)$ & $56.04(6.81)$ & 0.202 \\
T Conduct & $57.95(8.25)$ & $59.32(7.94)$ & 0.490 \\
\hline
\end{tabular}

* Independent sample t test

Table 5. Diagnosis among the participants $(n=126)$.

\begin{tabular}{|lll|}
\hline Diagnosis & Number & Percentage \\
Depression & 5 & 3.96 \\
Anxiety disorder & 11 & 8.73 \\
ADHD & 2 & 1.58 \\
ODD & 3 & 2.38 \\
Conduct disorder & 8 & 6.34 \\
Total & 29 & 23.01 \\
\hline
\end{tabular}

The common problem were anxiety $11(8.37 \%)$, conduct $8(6.34 \%)$, depression $5(3.96 \%)$ followed by ODD 3 $(2.38 \%)$, and ADHD $2(1.59 \%)$. (Table 6$)$ shows that females had more diagnosable problems than the male children [17 (24.63\%) Vs $12(21.05 \%)]$. Depression [5 $(7.24 \%)$ vs 0 ] and anxiety [9 (13.04\%)vs2 (3.50\%)] were over represented in females whereas ADHD [2 $(3.5 \%)$ vs 0] ODD [3 $(5.26 \%)$ vs 0 ] and conduct disorder [5 $(8.77 \%)$ vs3 $(4.34 \%)]$ were more common 
in male children. Finally, the prevalence of emotional and behavioural problem among our participants was $28.57 \%(26.31 \%$ in boys and $30.43 \%$ in girls) based on CBCL/6-18 years and $23.01 \%(21.05 \%$ in boys and $24.63 \%$ in girls) based on final diagnosis, as shown in (Table 7).

\begin{tabular}{|c|c|c|c|c|c|c|}
\hline & Affective & Anxiety & ADHD & ODD & Conduct & Total \\
\hline \multicolumn{7}{|l|}{ Gender } \\
\hline $\begin{array}{l}\text { Male } \\
\mathrm{N}=57\end{array}$ & 0 & $\begin{array}{l}2 \\
(3.50)\end{array}$ & $\begin{array}{l}2 \\
(3.50)\end{array}$ & $\begin{array}{l}3 \\
(5.26)\end{array}$ & $\begin{array}{l}5 \\
(8.77)\end{array}$ & $\begin{array}{l}12 \\
(21)\end{array}$ \\
\hline $\begin{array}{l}\text { Female } \\
\mathrm{N}=69\end{array}$ & $\begin{array}{l}5 \\
(7.24)\end{array}$ & $\begin{array}{l}9 \\
(13.04)\end{array}$ & 0 & 0 & $3(4.34)$ & $\begin{array}{l}17 \\
(24)\end{array}$ \\
\hline
\end{tabular}

\begin{tabular}{|lll|}
\hline \multicolumn{3}{|l|}{ Table 7. Distribution of problem based on gender. } \\
\hline Gender (n) & $\begin{array}{l}\text { Clinical range T } \\
\text { score } \mathbf{n}(\%)\end{array}$ & $\begin{array}{l}\text { Final diagnosis } \\
\mathbf{n}(\%)\end{array}$ \\
Male (57) & $15(26.31)$ & $12(21.05)$ \\
Female (69) & $21(30.43)$ & $17(24.63)$ \\
Total (126) & $36(28.57)$ & $29(23.01)$ \\
\hline
\end{tabular}

\section{DISCUSSION}

The aim of our study was to measure the emotional and behavioural problems in sheltered homeless children of Nepal. It has been stated that homeless children are three to four times more likely to experience emotional and behavioural problems than the general population, ${ }^{3}$ but this is not the case in this study. Despite the traumatic and demoralising experience of homelessness, the proportion of emotional and behavioural problem in our study is similar to that of general school going children as shown by the study done around 9 years back among school going children in Kathmandu. ${ }^{5}$ The study found that the prevalence of emotional and behavioural problems in Nepalese school going children of age group 4-18 using teacher version of CBCL was $27 \%(31.6 \%$ among boys and $22.4 \%$ among girls) which is similar to our finding where prevalence of clinical range $\mathrm{T}$ score in CBCL/6-18 was $28.57 \%$ (26.31\% in boys and $30.43 \%$ in girls). ${ }^{4}$ But, homeless children in our study have less problems than those with physically disabled children in similar context. ${ }^{6}$ Even few Indian studies in general school age children have shown similar results to ours. ${ }^{7}$

This finding is however similar to some studies in which also reported that $\mathrm{CBCL}$ scores are similar in homeless, previously homeless and never homeless children. ${ }^{4}$ Though, we did not find any difference in homeless and school children, our prevalence rate is similar to other similar studies. ${ }^{8}$ The observed finding in this study may be because of the protective effect of the shelter home where our study participants are kept, that takes care of the basic needs of the children including health and education.

But in contrast to the previous study, ${ }^{5}$ this study showed that the emotional and behavioural problems were more common in girls than boys $126.31 \%$ in boys vs $30.43 \%$ in girls). However, our findings agree with the well-established phenomenon that externalising problems are commoner in the boys and the internalising problems occurs more in the girls. Similarly, the prevalence of different disorders in our study is similar to the findings in the general children in the community. ${ }^{9}$ Several factors may be responsible for these variations. Some of these can be explained by differences in sample populations in the technique used in screening and diagnosis. Moreover, prevalence figure vary from culture to culture, within country and across countries, and the differences are often enormous.

Though this study is first of its kind in Nepal, there were few limitations. As for enrollment of the participants only one organization consented for the study working in this field. Other organizations did not give informed consent on behalf of their children. So, we included every child in only one of the shelters with its own criteria for admission. This shelter had kept only those children who were willing to stay and were motivated and there was good psychological as well as physical support for the children. This might result in underestimation of the real problem, as those with problems were already dealt by the psychologist in the shelter. This may weaken the generalization of the findings of this study. Nonetheless, it can be used as a pilot study which has provided an important indication that if one can provide shelter, education or take care of the basic needs of homeless children, it can reduce the burden of the psychological problems.

\section{CONCLUSIONS}

It is essential to understand the mental health issues among homeless children as poverty and homelessness has been shown to have negative effect in the psychological well-being of children. The prevalence of emotional and behavioural problem among the participants was $28.57 \%$ based on CBCL/6-18 years and $23.01 \%$ based on final diagnosis. This study found that the rate of emotional and behavioral disorder among homeless children is similar to the school aged non homeless children. These findings might be because of the possible positive role of shelter homes in the psychological health of the children.This study has highlighted the need of further interventions among these children and better studies in this field. 


\section{REFERENCES}

1. Hassan ZK. Children mental health problems and prospects using primary health care.Pak J Child Mental Health.1991.

2. Homelessness \& Poverty in America.National Law Center on Homelessness and Poverty. 2010 [cited 9 Sep]; Available from: http://www.nlchp.org/hapia.cfm.

3. Homeless families with children. Washington.National Coalition for the Homeless. August 2007 [cited 10 Sep]; Available from: URL: http: //www.nationalhomesless.org.

4. MenkeEM, Wagner JD.A comparative study of homeless, previously homeless, and never homeless school-aged children's health. IssuesComprPediatrNurs. 1998,Jul-Sep;20(3):153-73.
5. Adhikari SR. A study of the emotional and behavioural problem in school going children in Kathmandu. [Thesis]. [Maharajgunj]: Institute of Medicine; 2003.

6. Ojha SP. A study of the emotional and behavioural problem in physically disabled children. [Thesis].[Maharajgunj]: Institute of Medicine; 1999.

7. TR Deivasigamani.Psychiatric morbidity in primary school children - an epidemiological study.Indian J Psychiatry.1990 Jul-Sep;32(3):235-40.

8. Agustin MS, Cohen P, Rubin D, Cleary SD, Erickson CJ, Allen JK.The Montefiore community children's project: a controlled study of cognitive and emotional problems of homeless mothers and children.J Urban Health. 1999 Mar;76(1):39-50.

9. Peterson AC,Compas BE, Brooks-Gunn Jet al. Depression in adolescence.American Psychologist.1993;48(2):155-68. 